\title{
The pro-apoptosis and pro-inflammation role of LncRNA HIF1A- AS1 in Coxsackievirus B3-induced myocarditis via targeting miR-138
}

\author{
Huili Cao, Bin Yang, Yajing Zhao, Xingqiang Deng, Xiaoyu Shen \\ Department of Cardiology, The Second Hospital of Shanxi Medical University, Taiyuan, China \\ Contributions: (I) Conception and design: H Cao; (II) Administrative support: H Cao, B Yang; (III) Provision of study materials or patients: B Yang, \\ Y Zhao, X Deng; (IV) Collection and assembly of data: H Cao, B Yang; (V) Data analysis and interpretation: H Cao, Y Zhao, X Deng, X Shen; (VI) \\ Manuscript writing: All authors; (VII) Final approval of manuscript: All authors. \\ Correspondence to: Huili Cao. Department of Cardiology, The Second Hospital of Shanxi Medical University, No. 382 Wuyi Road, Xinghualing \\ District, Taiyuan 030001, China. Email: huilicao2000@outlook.com.
}

\begin{abstract}
Background: Cardiomyocyte apoptosis and inflammation induced by Coxsackievirus B3 (CVB3) are key pathogenetic mechanisms in viral myocarditis. Alterations in microRNAs and lncRNAs are associated with cardiac remodeling. However, whether the microRNA and lncRNA interact to regulate cardiomyocyte apoptosis and inflammation is unknown.

Methods: BALB/c mice were infected with CVB3 to generate acute viral myocarditis model. The expression levels of lncRNA HIF1A-AS1 and miR-138 were examined in mouse myocardium and primary cardiomyocytes with CVB3 infection. We then knocked down HIF1A-AS1 by siRNA and upregulated miR138 by microRNA mimics in cardiomyocytes.

Results: The expression of lncRNA HIF1A-AS1was significantly increased in CVB3-induced myocardium and cardiomyocytes. As documented by flow cytometry, silencing of HIF1A-AS1 alleviated late apoptosis $(5.1 \% \pm 2.8 \%$ vs. $17.2 \% \pm 4.2 \%, \mathrm{P}<0.01)$ and ROS production $(68.73 \% \pm 2.78 \%$ vs. $90.40 \% \pm 2.86 \%, \mathrm{P}<0.01)$ compared to their levels in cardiomyocytes transfected with control siRNA. The content of proinflammatory cytokines was substantially decreased by HIF1A-AS1 siRNA. Furthermore, we identified that HIF1A-AS1 bound to miR-138 and significantly suppressed miR-138 expression. Blocking HIF1A-AS1 attenuated IкB $\alpha$ phosphorylation and NF- $\mathrm{B}$ activity, while cotransfection with miR-138 mimics markedly reversed its effect. Conclusions: In conclusion, lncRNA HIF1A-AS1 promotes NF- $\kappa$ B signaling and subsequently aggravates cardiomyocyte apoptosis and inflammation via targeting miR-138.
\end{abstract}

Keywords: LncRNA HIF1A-AS1; miR-138; apoptosis; inflammation; viral myocarditis

Submitted May 31, 2020. Accepted for publication Aug 30, 2020.

doi: $10.21037 / \mathrm{cdt}-20-545$

View this article at: http://dx.doi.org/10.21037/cdt-20-545

\section{Introduction}

Myocarditis is a prevalent cause for inflammatory heart diseases that can progress to dilated cardiomyopathy and heart failure in children and young adults (1). Common causal pathogens for myocarditis worldwide are enteroviruses, such as parvovirus B19, Borrelia burgdorferi and coxsackievirus B3 (CVB3) (2). Clinical trials have reported elevated levels of circulating tumor necrosis factor- $\alpha$ (TNF- $\alpha$ ), interleukin-1 $\beta$ (IL-1 $\beta$ ), IL-6 and other pro-inflammatory cytokines in patients with myocarditis $(3,4)$. However, to date, the pathogenesis of viral myocarditis remains unclear. During viral infection, cytokine exposure and robust inflammatory response 
induces the generation of reactive oxygen species (ROS) and excessive apoptosis in cardiac cells $(4,5)$. It is, therefore, conceivable that modulation of inflammation and suppression of cardiac apoptosis are potential therapeutic strategies for CVB3-induced myocarditis. Furthermore, in response to $\mathrm{CVB} 3$ infection and subsequent inflammation, several potent signaling pathways are profoundly altered, such as NF- $\kappa$ B and STAT3 pathways $(6,7)$.

Over past decades, a number of studies demonstrated the critical roles of miRNAs and lncRNAs in cardiac hypertrophy and heart failure $(8,9)$. Among these studies, miR-221 and miR-222 were increased in myocardium during cardiac remodeling after acute viral myocarditis (10). Concurrently, inhibition of miR-221/miR-222 and miR-214 alleviated inflammation and cardiac injury during CVB3induced myocarditis.

On the other hand, lncRNAs are important regulators of gene expression in a wide range of development processes and diseases $(11,12)$. LncRNA HIF1A-AS1 was highly expressed in the maternal allele and acted as an oncogene via inhibition of HIF- $1 \alpha$ and cell cycle in some types of cancer $(13,14)$. However, the expression and role of HIF1AAS1 in cardiac remodeling remains unclear.

In this study, we first detected whether HIF1AAS1 was involved in the process of CVB3-induced myocarditis. Additionally, the role of HIF1A-AS1 in myocytic inflammation and apoptosis within the mouse model of CVB3-induced myocarditis was explored. We present the following article in accordance with the ARRIVE reporting checklist (available at http://dx.doi. org/10.21037/cdt-20-545).

\section{Methods}

\section{Virus and animals}

All animal experiments were performed under a project license (No. 2018-1327) granted by Institute Animal care and Use Committee of The Second Hospital of Shanxi Medical University for the care and use of animals. Eightweek-old male BALB/c mice were obtained from the Shanghai SLAC Laboratory Animal Co, Ltd. (Shanghai SLAC Laboratory Animal Co Ltd., Shanghai, China) and housed in specific pathogen-free cages with well-ventilated stainless-steel grid tops at room temperature with a 12 hour light/dark cycle. CVB3 (Nancy strain) was maintained by passage through Hela cells (ATCC number: CCL-2). Mice were infected with CVB3 by an intraperitoneal (i.p.) injection with $10^{3} \mathrm{PFU}$ doses of the virus. At different time points, experimental mice were sacrificed. The heart was excised while still beating and perfused with PBS to move blood from myocardium. Paraffin sections of each sample were prepared and collagen was observed by Masson's trichrome staining following standard procedures. Additionally, paraffin sections were stained with fluorescein isothiocyanate-conjugated wheat germ agglutinin (WGA, REAGEN Incorporation, catalog FT-sj80190, China) to confirm the size of cardiomyocytes.

\section{Cell culture and infection}

Neonatal mouse cardiomyocytes were isolated as previously described (7). Briefly, the hearts obtained from 1-3 days $\mathrm{BALB} / \mathrm{c}$ mice were removed rapidly into cold Hanks' balanced salt solution (HBSS). After washing and mincing, tissues were digested in $0.05 \%$ trypsin (GIBCO, USA) for $30 \mathrm{~min}$ at $4{ }^{\circ} \mathrm{C}$ with rotation. The tissues were transferred into DMEM (GIBCO, USA) containing 20\% FBS (Fetal bovine serum, Gibco) to terminate the digestion. The supernatant containing released cells was removed every 10 min into an equal volume of cold DMEM complete medium (abandon the first two times) until most of the myocardium was disrupted. Cells were collected and enriched by two sequential pre-plating steps on $90-\mathrm{mm}$ dishes which removed non-cardiomyocytes. The isolated cells were incubated with $5 \% \mathrm{CO}_{2}$ at $37{ }^{\circ} \mathrm{C}$ for $1 \mathrm{~h}$. The unattached cardiomyocytes were seeded into fibronectin-coated 12 well tissue culture plates (Costar, USA) and subsequent experiments were performed when the cardiomyocytes formed a confluent monolayer and beat in synchrony at $72 \mathrm{~h}$.

To explore the effects of CVB3 on cardiomyocytes, neonatal cardiomyocytes $\left(2 \times 10^{6} /\right.$ well $)$ were infected with 10 MOI of CVB3 (Nancy strain) diluted in DMEM without any supplements. After 2 hours, virus was removed and cells were washed twice with PBS. Afterwards, cells were incubated in starving medium for additional 48 hours. Control cells were treated equally in the absence of virus.

\section{Cell transfection}

Th e s i R N A a g a i n s t H I F 1 A - S 1 (5'-GUCAAUUGGUUGAUCACCCG-3') and scramble siRNA (5'-UUCUCCGAACGUGUCACGUTT-3') were constructed by GenePharma Corporation (Shanghai, China). The miR-138 mimics and control mimics were also purchased from GenePharma Corporation. Cardiomyocytes were transfected with siRNAs (100 $\mathrm{nM}$ ) using RNAiMax 
(ThermoFisher Scientific, catalog 13778150, USA) according to the manufacturer's instructions.

\section{ELISA measurements}

The levels of IL-1 $\beta$ (R\&D, catalog MLB00C, USA), IL-6 (R\&D, catalog M6000B, USA) and TNF $\alpha$ (abcam, catalog ab212073, USA) in cell culture supernatants were determined by ELISA. The contents of these cytokines were quantified by reading absorbance in an automatic microplate reader (SpectraMax i3, Molecular Devices, USA) and calculating from a standard curve.

\section{Cell apoptosis analysis}

The apoptosis of cardiomyocytes was determined by Annexin V-FITC-PI staining (BD Biosciences, catalog 556547, USA) and analyzed by flow cytometry (FACSCalibur, BD Biosciences, USA) as described in the previous study (6). Annexin $\mathrm{V}$ positive cells were considered as early apoptosis, while cells stained by Annexin V and PI were considered as late apoptosis.

\section{ROS assay}

ROS production in CVB3-induced cardiomyocytes was quantified using the fluorescent probe 2', 7'-dichlorofluorescein diacetate (DCFH-DA, catalog C2938, Thermo Scientific, USA). After CVB3 infection and siRNA transfection, primary cardiomyocytes $\left(5 \times 10^{5} /\right.$ well $)$ were labeled with $0.5 \mathrm{nM}$ DCFH-DA for $30 \mathrm{~min}$ at $37^{\circ} \mathrm{C}$. Cells were collected and washed by PBS. The fluorescence intensity of ROS was measured by flow cytometry.

\section{Western blot analysis}

Cells were lysed in RIPA lysis buffer and phenylmethanesulfonyl fluoride at $4{ }^{\circ} \mathrm{C}$ for $30 \mathrm{~min}$. Mitochondria were isolated using the Mitochondria Isolation Kit for Cultured Cells (Thermo Scientific, catalog 89874, USA) and prepared for western blot analysis. A total of $30 \mu \mathrm{g}$ of protein was separated in a $6 \%$ to $12.5 \%$ SDS-PAGE gel, transferred to a PVDF membrane and probed with the following antibodies overnight at $4{ }^{\circ} \mathrm{C}$ : $\mathrm{p}-\mathrm{I} \kappa \mathrm{B} \alpha(1: 1,000$ dilution, Cell Signaling Technology, catalog 5209, USA) and t-IкB $\alpha(1: 1,000$ dilution, Cell Signaling Technology, catalog 4814, USA). After incubation with either HRP-conjugated anti-rabbit or anti-mouse antibodies, immunoreactive bands were visualized by chemiluminescence (ECL, Pierce, USA), and the membranes were exposed to light film. The GADPH antibody (1:2,000 dilution; Santa Cruz, catalog sc-137179, USA) was used as a loading control.

\section{RNA extraction and $q R T-P C R$ analysis}

Total RNA was isolated from mouse heart tissue using the RNAprep pure Tissue kit (Tiangen, catalog DP431, China). Total RNA from neonatal cardiomyocytes was extracted using Trizol reagent (Invitrogen, USA). Reverse transcription was performed using $1 \mu \mathrm{g}$ of RNA and PrimeScript RT reagent Kit (Takara, catalog RR036A, Japan). Quantitative RT-PCR was performed using a LightCycler 96 Real-Time PCR System (Roche Diagnosis, USA) using FastStart Essential DNA Green Master assay (Roche, catalog 06402712001, USA). The $2^{-\Delta \Delta C t}$ method was used for semi-quantitative analysis. All results were normalized against $\beta$-actin as an internal standard. Primers are listed in Table 1.

\section{Dual-luciferase reporter assay}

$293 \mathrm{~T}$ cells were maintained in DMEM supplemented with $10 \%$ fetal bovine serum. To determine whether lncRNA HIF1A-AS1 could bind to the miR-138, 293T cells were seeded in 24-well plates and co-transfected using FuGENE 6 (Promega, catalog E2691, USA) with $10 \mathrm{nM}$ miR-138 mimic or control miRNA mimic and $2.5 \mu \mathrm{g}$ pGL3 vector containing putative fragments of the HIF1A-AS1. After $48 \mathrm{~h}$ of transfection, the relative luciferase expression normalized to Renilla activity was measured using an automatic micro-plate reader with the dual-luciferase reporter assay (Promega, catalog E1960, USA).

\section{Immunofluorescence staining}

After being incubated for regular time, primary cardiomyocytes were fixed in $4 \%$ paraformaldehyde and permeabilized with $0.1 \%$ Triton $\mathrm{X}-100$ at room temperature for $10 \mathrm{~min}$ respectively. Thereafter, cells were incubated with p65 antibody (diluted 1:50, Abcam, catalog ab16502, USA) and further stained with appropriate FITCconjugated secondary antibodies (Santa Cruz, catalog sc65218, USA). Nucleus was counterstained with DAPI. Confocal microscopy was performed with the Confocal Laser Scanning Microscope System (Zeiss, Germany). 
Table 1 Summary of primer sequences used for RT-PCR

\begin{tabular}{ll}
\hline Gene & Sequence \\
\hline HIF1A-AS1 & Forward: 5'-AGGATGGAACTATCTGGG-3' \\
& Reverse: 5'-AAGTTAAAGGGAGAAGCTA-3' \\
TNF- $\alpha$ & Forward: 5'-CCCTCACACTCAGATCATCTTCT-3' \\
& Reverse: 5'-GCTACGACGTGGGCTACAG-3' \\
IL-6 & Forward: 5'-TAGTCCTTCCTACCCCAATTTCC-3' \\
& Reverse: 5'-TTGGTCCTTAGCCACTCCTTC-3' \\
IL-1 $\beta$ & Forward: 5'-GCCTCGTGCTGTCGGACC-3' \\
& Reverse: 5'-TGTCGTTGCTTGGTTCTCCTTG-3' \\
$\beta$-actin & Forward: 5'-GGCTGTATTCCCCTCCATCG-3' \\
& Reverse: 5'-CCAGTTGGTAACAATGCCATGT-3'
\end{tabular}

\section{Statistical analysis}

Data were expressed as mean \pm SEM. Statistical analysis was performed with SPSS 14.0 software (SPSS Inc., Chicago, IL, USA). Independent two group's analyses were performed by Student $t$-test. Three or more groups were compared by oneway ANOVA and post-hoc analysis with the Bonferroni test. Statistical significance was defined as $\mathrm{P}<0.05$.

\section{Results}

\section{LncRNA HIF1A-AS1 is increased in CVB3-induced myocarditis}

To investigate the association between HIF1A-AS1 and myocarditis, we initially explored the expression of HIF1A-AS1 in myocardium after 3-, 5- and 7-day CVB3 infection. At 7-day of CVB3-induced viral myocarditis, collagen was moderately accumulated and cardiomyocytes were larger as compared to control group (Figure 1A,B). With the progression of acute viral myocarditis, quantitative RT-PCR revealed that the expression levels of lncRNA HIF1A-AS1 were gradually increased (Day 1 $1.17 \pm 0.25$ vs. Day $74.37 \pm 0.33, \mathrm{P}<0.01$ ) (Figure $1 C$ ). Likewise, it is critical to study the effects of CVB3 on HIF1A-AS1 expression in cardiomyocytes. The expression levels of HIF1AAS1 were time-dependently increased and reached its peak after 7-day CVB3 infection (Figure 1D).

\section{Reduced lncRNA HIF1A-AS1 attenuates oxidative stress and apoptosis of cardiomyocytes induced by CVB 3 infection}

To determine the biological functions of HIF1A-AS1 in cardiomyocytes, we first knocked down HIF1A-AS1 using HIF1A-AS1 siRNA. The expression of HIF1AAS19 was downregulated by $76.3 \%$ (Figure $2 A$ ). It is widely accepted that oxidative stress of cardiomyocytes is involved in the pathogenesis and development of viral myocarditis. Therefore, we examined the effect of HIF1A-AS1 on oxidative stress by flow cytometry and found that knockdown of HIF1A-AS1 suppressed ROS production after 3 -day CVB3 infection evaluated $(90.40 \% \pm 2.86 \%$ vs. $68.73 \% \pm 2.78 \%, \mathrm{P}<0.01$ ) (Figure $2 B, C$ ). Next, we investigated whether HIF1A-AS1 played a pivotal role in cardiomyocyte apoptosis during CVB3 infection. Cell apoptosis was assessed by Annexin V and PI double staining followed by flow cytometry. As shown in Figure 2D,E, depletion of HIF1A-AS1 markedly inhibited the late apoptosis of cardiomyocytes induced by CVB3 infection for 3 days $(17.2 \% \pm 4.2 \%$ vs. $5.1 \% \pm 2.8 \%, \mathrm{P}<0.01)$, whereas it did not significantly influence the early apoptosis ( $45.3 \% \pm 4.7 \%$ vs. $32.6 \% \pm 6.4 \%, \mathrm{P}=0.205$ ).

\section{Reduced lncRNA HIF1A-AS1 alleviates inflammatory response of cardiomyocytes induced by $C V B 3$ infection}

Recent studies have demonstrated that excessive inflammation induced by CVB3 infection is substantially involved in the pathogenesis of myocarditis and work via oxidative stress and apoptosis of cardiomyocytes $(4,15)$. In this regard, we identified that knockdown of HIF1AAS1 remarkably inhibited the mRNA expression of TNF $\alpha$, IL- $1 \beta$ and IL- 6 in cardiomyocytes induced by CVB3 infection for 3 days $(\mathrm{P}<0.01)$ (Figure $3 A, B, C)$. Furthermore, the cell supernatants were collected and the cytokine levels were measured by ELISA. Accordingly, compared to siCTR group, the production of TNF $\alpha(37.6 \pm 4.8 \mathrm{vs}$. $11.3 \pm 2.5 \mathrm{pg} / \mathrm{mL}, \mathrm{P}<0.01)$ and $\mathrm{IL}-6(29.7 \pm 5.4 v s .11 .8 \pm 3.0 \mathrm{pg} / \mathrm{mL}$, $\mathrm{P}<0.01)$ were significantly decreased in cell supernatants from siHIF1A-AS1-treated cardiomyocytes (Figure 3D,E), whereas the secretion of IL- $1 \beta$ was unchanged $(\mathrm{P}=0.482)$.

\section{LncRNA HIF1A-AS1 is bound to miR-138 and suppresses miR-138 expression}

It was previously reported that HIF1A-AS1 regulated tumor progression via interaction with diverse microRNAs, including miR-675, miR-29b, miR-204 and miR-138 (16-19). Inspired by these phenomena, we initially explored the expression levels of these candidate microRNAs during acute viral myocarditis. As shown in Figure $4 A$, as 
A

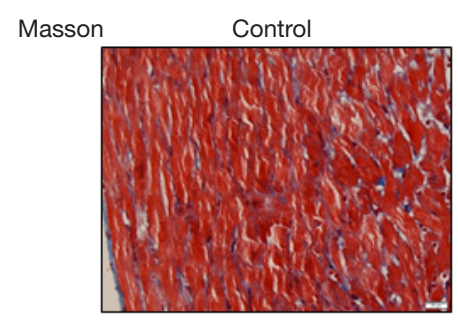

CVB3 7 days

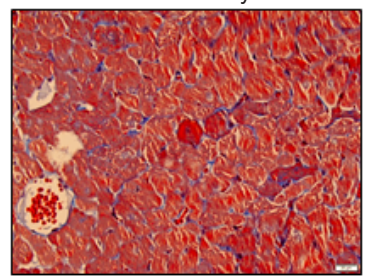

C

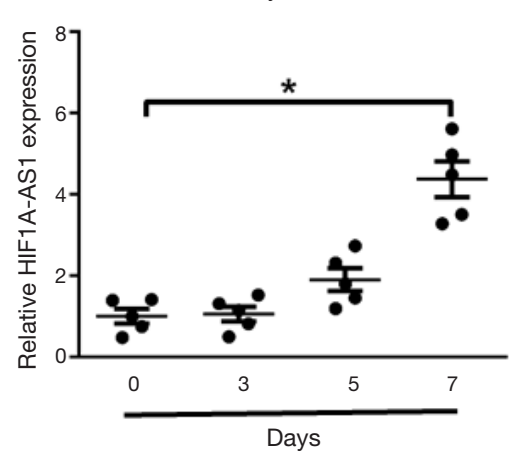

B

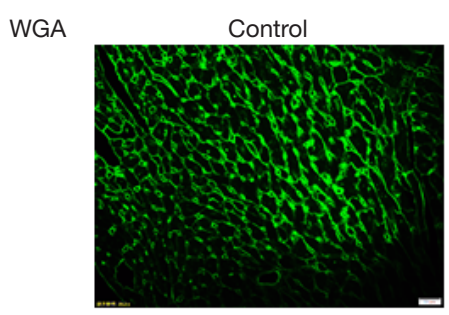

CVB3 7 days

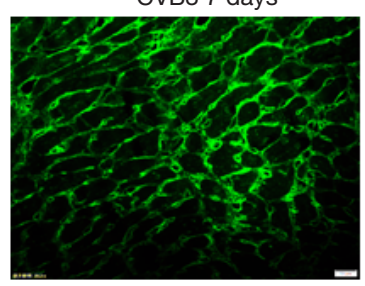

Cardiomyocytes

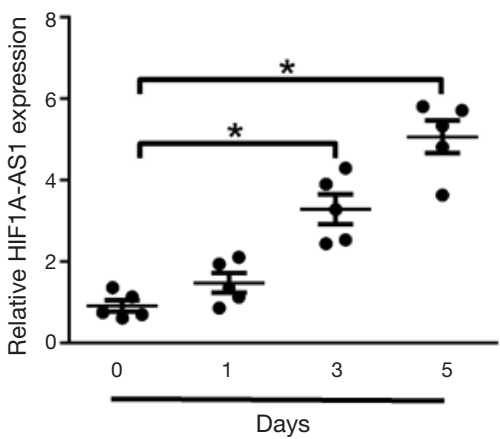

Figure 1 LncRNA HIF1A-AS1 is upregulated in myocardium and cardiomyocytes induced by CVB3. (A) Masson staining showed the morphology of left ventricular at 7-day of CVB3-induced viral myocarditis. (B) Wheat germ agglutinin (WGA) staining of left ventricular at 7-day of CVB3-induced viral myocarditis. (C,D) The expression levels of HIF1A-AS1 were measured by quantitative RT-PCR in mouse myocardial tissue $(\mathrm{C})$ and primary cardiomyocytes $(\mathrm{D})$ treated with CVB3 at the indicated time point. Data are mean \pm SEM and representative of three separate experiments. * $\mathrm{P}<0.05$.

compared to control myocardium, the expression level of miR-138 was downregulated in myocardium infected by CVB3 for 7 days, while there were no significant differences in the expression levels of miR-675, miR$29 \mathrm{~b}$ and miR-204 between control cardiomyocytes and CVB3-induced myocarditis (Figure 4A). Based on established sequences derived from the database results (http://genome.ucsc.edu/), we hypothesized that the target of HIF1A-AS1 linked with the inflammation and apoptosis was miR-138 and examined the expression level of miR138. As a result, the expression of miR-138 was markedly decreased by HIF1A-AS1 silencing (Figure 4B). In order to determine the potential fragment of HIF1A-AS1 interacted with miR-138, the candidate sequences of HIF1AAS1 (chr14:61,681,041-chr14:61,695,823), which are consensus-binding sequences of miR-138, were amplified and inserted downstream of the luciferase reporter gene in the pGL3 vector to construct Luc-HIF1A-AS1 vector. The result indicated that cotransfection of miR-138 mimics with Luc-HIF1A-AS1 in 293 T cells led to $67.3 \%$ inhibition of luciferase activity compared to samples with control miRNA mimics (Figure 4C,D).

\section{LncRNA HIF1A-AS1 attenuates NF- $\kappa$ B signaling via inbibition of miR-138}

To elucidate the mechanism underlying HIF1A-AS1miR-138 mediated apoptosis and inflammation of cardiomyocytes stimulated by CVB 3 infection, we studied the role of HIF1A-AS1 and miR-138 on NF- $\mathrm{BB}$ 
A

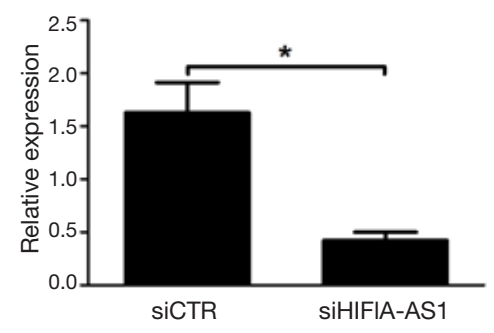

C

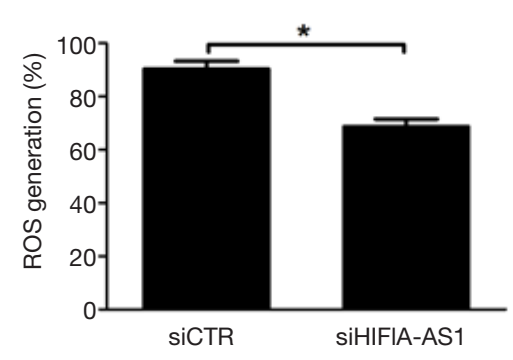

E

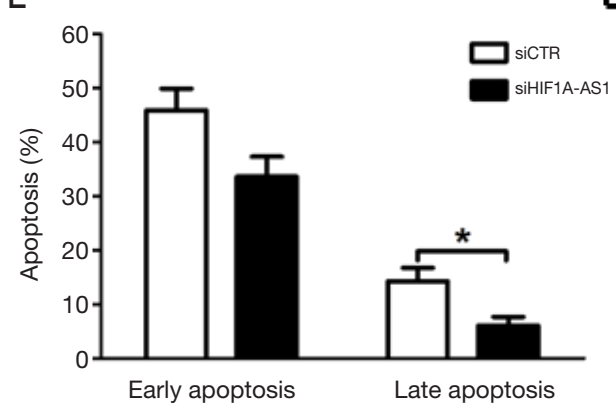

B
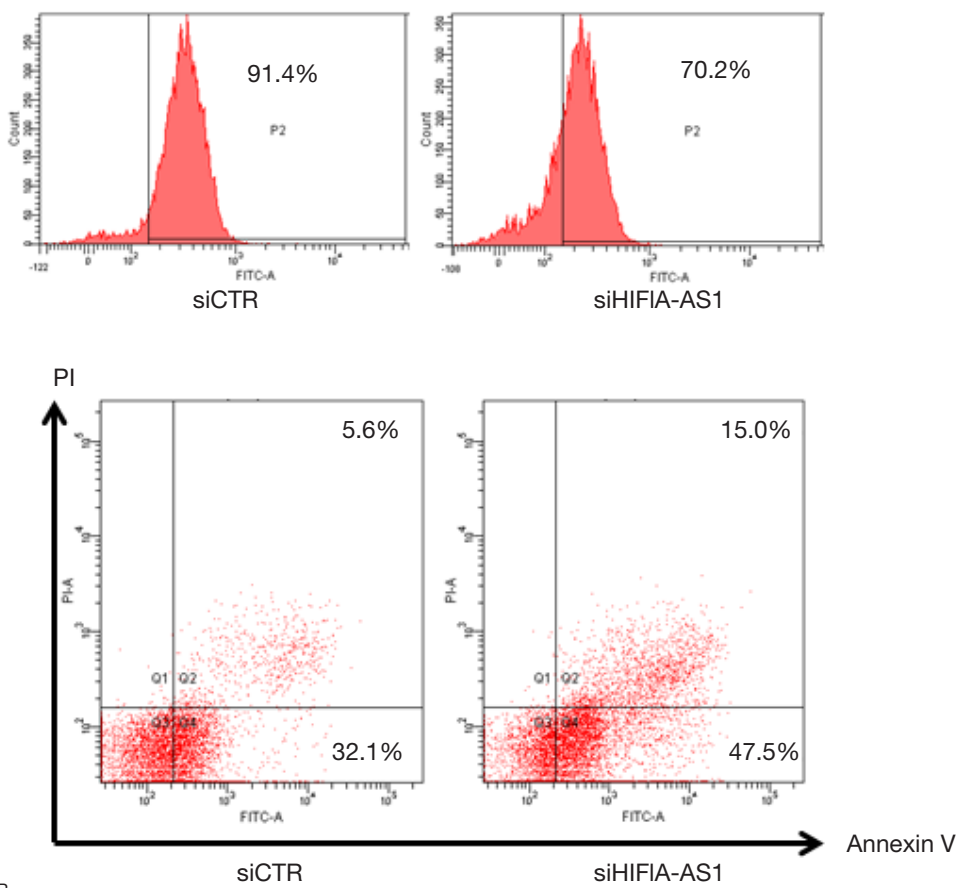

Figure 2 Knockdown of HIF1A-AS1 attenuates oxidative stress and apoptosis of cardiomyocytes treated with CVB3. (A) The efficiency of HIF1A-AS1 knockdown by siRNA was determined by quantitative RT-PCR. (B) Flow cytometry was performed to illustrate the role of HIF1A-AS1 depletion on reactive oxygen species (ROS) production in response to CVB3 infection, which reflected the severity of intracellular oxidative stress. (C) Statistical results for the percentage of ROS production were obtained by flow cytometry analysis. (D,E) The early and late apoptosis ratio of primary cardiomyocytes transfected with control siRNA and HIF1A-AS1 siRNA was analyzed by Annexin V-PI assay and flow cytometry. Mouse cardiomyocytes were transfected with control siRNA and HIF1A-AS1 siRNA for 48 hours and then stimulated by CVB3 for 3 days. Data are mean \pm SEM and representative of three separate experiments. ${ }^{*}, \mathrm{P}<0.05$. ROS, reactive oxygen species; siCTR, control siRNA.

pathway, which is referred as an established signaling for inflammation and apoptosis during acute viral myocarditis. As shown in Figure $5 A, B$, silencing of HIF1A-AS1

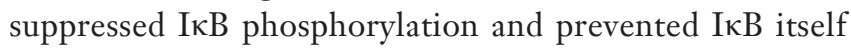
degradation in cardiomyocytes treated with CVB3 for $10 \mathrm{~min}$. However, the inhibition of IкB phosphorylation was substantially reversed by overexpression of miR138 in cardiomyocytes treated with CVB3. Consistently, silencing of HIF1A-AS1 blocked p65 nuclear translocation detected by confocal assay (Figure 5C) and mitigated NF- $\mathrm{kB}$ activation measured by luciferase activity relative to that in siCTR-treated $293 \mathrm{~T}$ cells, while cotransfection of miR-138 mimics could reverse NF- $\mathrm{KB}$ activity (Figure $5 D$ ). These results suggest that lncRNA HIF1A-AS1 inhibits NF- $\kappa \mathrm{B}$ activity through suppression of miR-138 in response to CVB3 infection. 
A

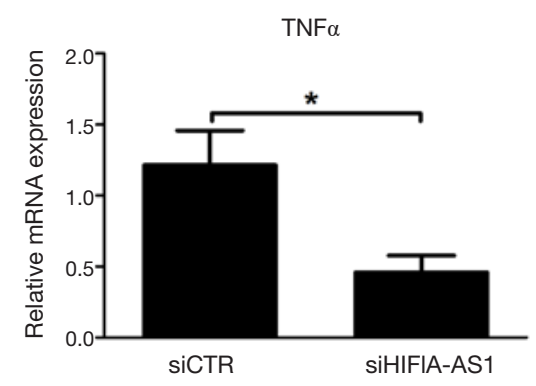

D

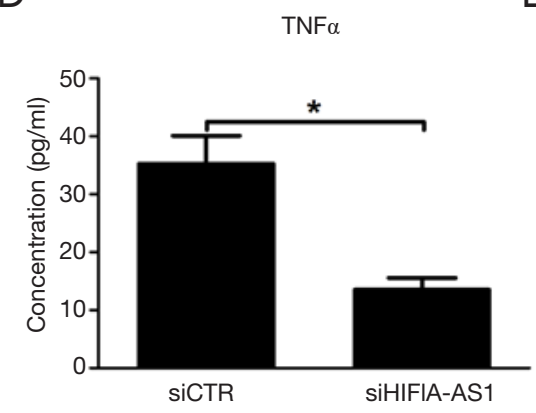

B

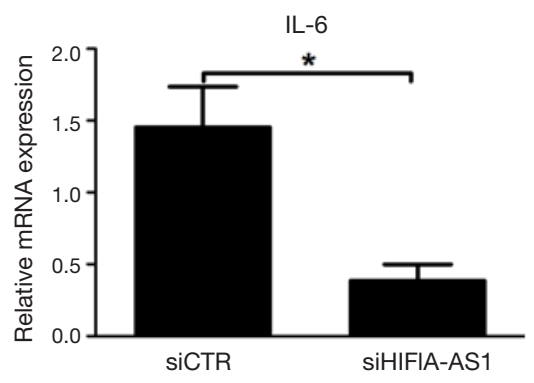

$E$

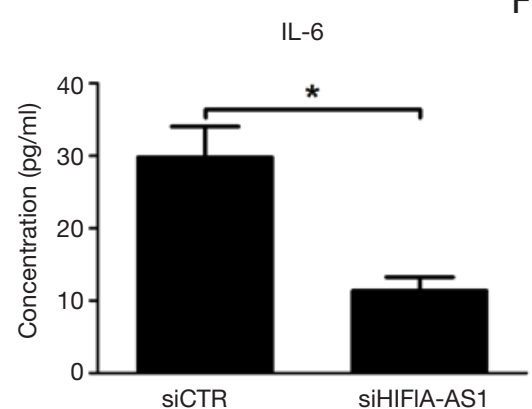

C

$\mathrm{F}$
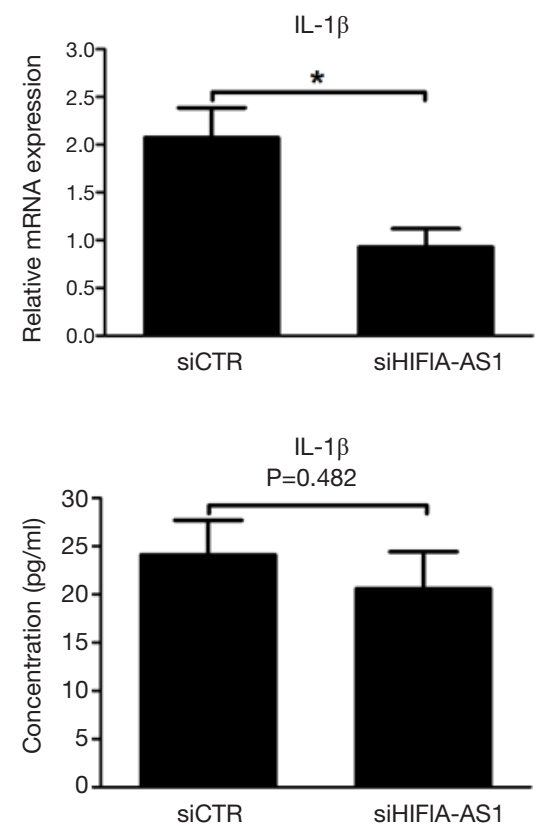

Figure 3 Knockdown of HIF1A-AS1 reduced inflammatory response of cardiomyocytes treated with CVB3. (A-C) Knockdown of HIF1AAS1 reduced the expression of TNF $\alpha$, IL-6 and IL-1 $\beta$ in cardiomyocytes treated with CVB3. (D-F) Knockdown of HIF1A-AS1 alleviated the secretion of TNF $\alpha$, IL-6 and IL-1 $\beta$ from cardiomyocytes treated with CVB3. Data are mean \pm SEM and representative of three separate experiments. *, $\mathrm{P}<0.05$.

\section{Discussion}

In the present study, we observed lncRNA HIF1A-AS1 and miR-138 alterations in mouse myocardium and primary cardiomyocytes exposed to CVB3. Silencing HIF1A-AS1 strongly repressed CVB3-induced cardiomyocyte apoptosis and inflammation, indicating a potential role of HIF1AAS1 in guiding the progression of acute viral myocarditis. In addition, the activation of NF- $\mathrm{KB}$ signaling could be the direct result from the interaction of HIF1A-AS1 with miR138. The study highlighted the importance of lncRNA HIF1A-AS1 in regulating apoptosis and inflammation in myocardium under the induction of CVB3.

LncRNAs are considered as transcribed RNA molecules over 200 nucleotides in length but are non-protein-coding. It is known that lncRNAs are capable of binding other RNA/DNA species as well as proteins and may thereby mediate processes at all stages from gene transcription and translation to protein function $(11,20)$. The involvement of lncRNAs in the pathogenesis of cardiac remodeling has been intensely investigated (9). In vitro and in vivo experiments have shown that noncoding RNAs, including lncRNAs and miRNAs, regulate the progression of CVB3-induced myocarditis $(9,21,22)$. Other studies from high-throughput screening and global lncRNA expression profiling have identified some altered lncRNAs in myocardial tissue during cardiac remodeling $(23,24)$. Viereck et al. found that lncRNA Chast accumulated in hypertrophic myocardial tissue and accelerated cardiomyocyte autophagy via negative regulation of coding protein Pleckstrin homology domaincontaining protein family $M$ member 1 (23). LncRNA Chaer also directly interacted with PRC2, consequently resulting in histone modification and aggravating cardiac hypertrophy (24). Conversely, lncRNA ROR was increased in hypertrophic cardiomyocytes and interfered with another noncoding RNA miR-133, which in turn aggravated cardiac remodeling (25). These findings suggest that the functions and mechanisms of these abnormal lncRNAs are diverse from each other.

In the present study, we revealed that IncRNA HIF1AAS1 was increased in CVB3-induced mouse myocardium and primary cardiomyocytes, and application of HIF1AAS1 siRNA attenuated the apoptosis and inflammation of cardiomyocytes. Numerous studies have identified that HIF1A-AS1 are differentially expressed in tumor 
A
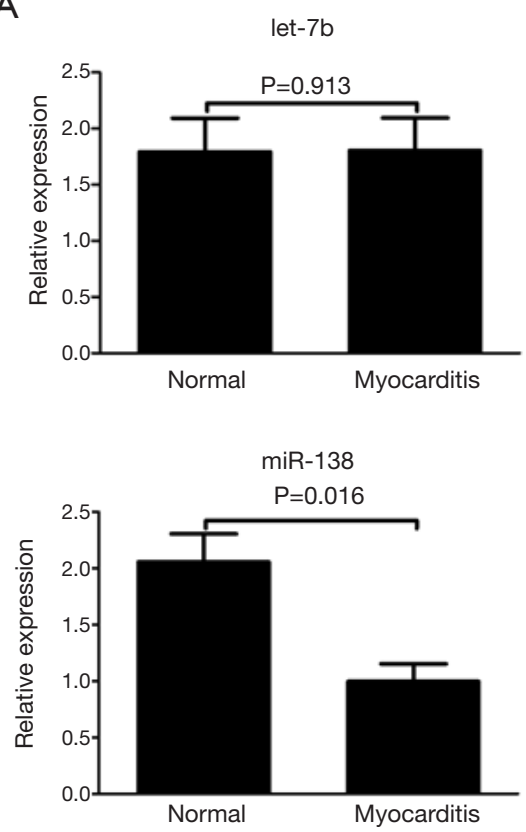

D

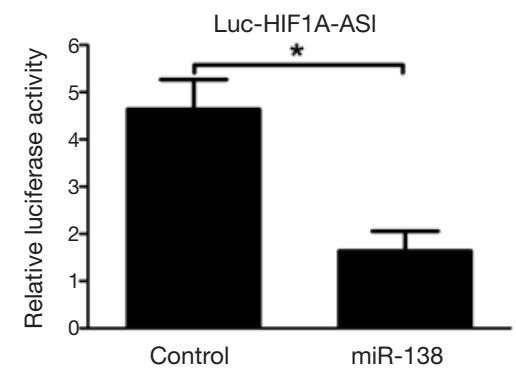

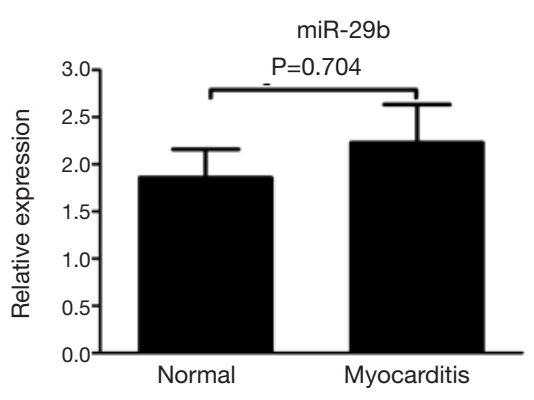

B

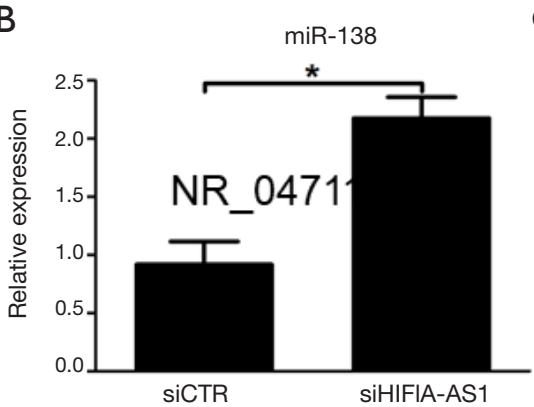

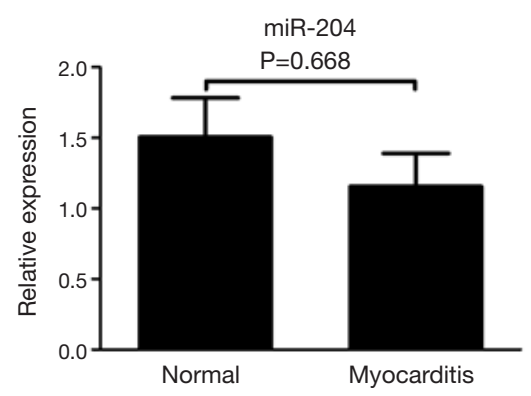

C

HIF1A-AS1 (NR_047116)

TCGCCCC - CACACTTAACT

III II IIIIIII

AGCUGGUGUUGUGAAUCAGGCCG

Figure 4 LncRNA HIF1A-AS1 interacted with miR-138 and suppressed its expression. (A) The expression levels of miR-675, miR-29b, miR-200a and miR-138 were measured by quantitative RT-PCR in normal myocardial tissue and myocardium infected by CVB3 for 7 days. (B) Knockdown of HIF1A-AS1 upregulated miR-138 expression evaluated by quantitative RT-PCR. (C) Predicted binding sites of lncRNA HIF1A-AS1 with miR-138 sequence. (D) Transfection with miR-138 mimics abolished the luciferase activity of pGL3 vector containing HIF1A-AS1 fragment (chr11: 2,016,882-chr11: 2,016,889). Data are mean \pm SEM and representative of three separate experiments. *, $\mathrm{P}<0.05$. siCTR, control siRNA.

progression and vascular remodeling as well (13,16-19,26). The increase in HIF1A-AS1 expression was reported to be associated with a poor prognosis in various cancers $(13,17)$. Similarly, HIF1A-AS1 was substantially upregulated during atherosclerosis (26). Nevertheless, the exact function of HIF1A-AS1 in viral myocarditis remains controversial. While Wang et al. (26) reported that HIF1A-AS1 served as a negative regulator for atherosclerosis through regulating inflammation, Acun et al. (27) indicated that inhibition of HIF1A-AS1 attenuated cell apoptosis and improved oxidative stress via mediating HIF-1 $\alpha$. In line with this observation, Wang et al. (28) found that depletion of HIF1A-AS1 markedly prevented endothelial cell apoptosis and death in vitro, suggesting HIF1A-AS1 as a potential therapeutic target of ischemic stroke. In fact, ROS activation resulted from CVB3 infection contributed to cardiomyocyte apoptosis and inflammatory response, affecting the fate of cardiomyocytes and impelling the proceeding of cardiac remodeling. Together, these data strongly supported the idea that HIF1A-AS1 could act 
A

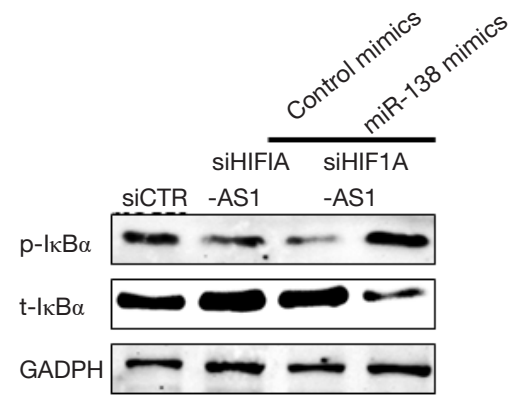

C

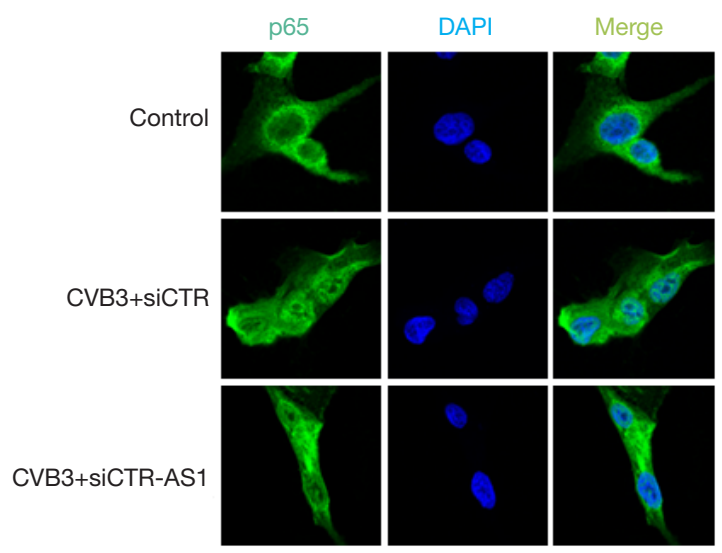

B

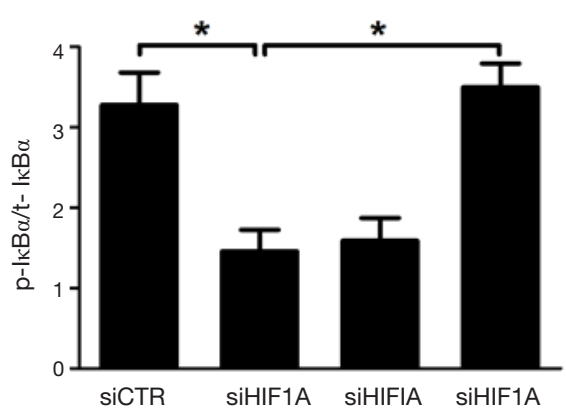

D

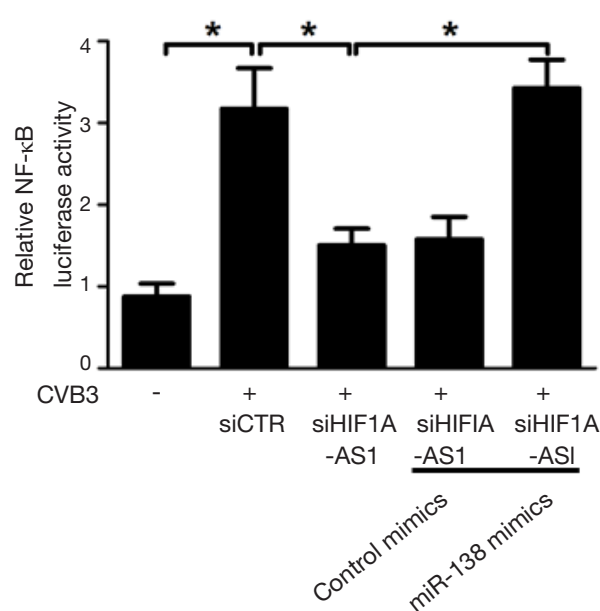

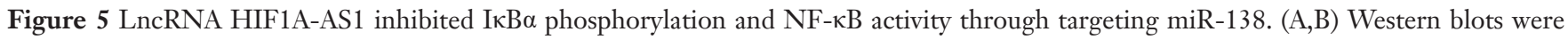
performed to evaluate the effect of HIF1A-AS1 and miR-138 on IKB $\alpha$ phosphorylation in cardiomyocytes treated with CVB3 for $10 \mathrm{~min}$. Data were calculated and presented as the density of each band. (C) Effect of HIF1A-AS1 on p65 nuclear translocation in cardiomyocytes treated with CVB3 was determined by confocal assay. (D) Effect of HIF1A-AS1 and miR-138 on NF-кB activity was measured by dualLuciferase assay. The pGL4.32 [luc2P/NF-kB-RE/Hygro] vector was cotransfected with HIF1A-AS1 siRNA and/or miR-138 mimics into 293 T cells for 48 hours using Lipofectamine 2000, and then NF-кB luciferase activity was measured using dual-luciferase assay. Data are mean \pm SEM and representative of three separate experiments. *, $\mathrm{P}<0.05$.

as one of lncRNAs mediating cardiac remodeling in viral myocarditis.

To gain insight into the underlying mechanisms of HIF1A-AS1 in CVB3-induced myocarditis, we reviewed the previous studies and detected the decreased expression of miR-138 in in vitro model of viral myocarditis. Mechanistically, HIF1A-AS1 directly interacted with miR-138 and suppressed miR-138 expression. Current studies have demonstrated that HIF1A-AS1 regulated the expression of downstream proteins and the signaling transduction by its interaction with microRNAs (16-19). In support of this notion, accumulating evidences have suggested that HIF1A-AS1 widely contributed to the pathogenesis of various diseases through the inhibition of miR-204 $(17,29,30)$. Nevertheless, our in vivo data failed to detect significant fluctuation of miR-204 in myocardium receiving $\mathrm{CVB} 3$ infection. In contrast, miR-138 was downregulated in CVB3-induced myocardial tissue and likely to be inversely associated with the expression of HIF1A-AS1. At present, little is known regarding HIF1AAS1 function as miR-138 sponge. To date, two relevant studies showed that HIF1A-AS1/miR-138 axis promoted migration, invasion as well as epithelial to mesenchymal transition via different downstream genes in colorectal 
cancer $(14,19)$. In agreement with these observations, our studies found that HIF1A-AS1 functioned as a precursor of miR-138 to post-translationally control downstream genes expression and was capable of activating NF- $\mathrm{B}$ activity, which eventually modulated cardiomyocyte apoptosis and inflammation in response to CVB3 infection.

\section{Conclusions}

Taken together, our observations suggested that HIF1AAS1/miR-138 axis is an important regulatory point in cardiomyocyte apoptosis and inflammation induced by CVB3 infection. Inhibition of HIF1A-AS1 may represent a promising therapeutic strategy for CVB3-mediated viral myocarditis.

\section{Acknowledgments}

Funding: None.

\section{Footnote}

Reporting Checklist: The authors present the study in accordance with the ARRIVE reporting checklist. Available at http://dx.doi.org/10.21037/cdt-20-545

Data Sharing Statement: Available at http://dx.doi. org/10.21037/cdt-20-545

Conflicts of Interest: All authors have completed the ICMJE uniform disclosure form (available at http://dx.doi. org/10.21037/cdt-20-545). The authors have no conflicts of interest to declare.

Ethical Statement: The authors are accountable for all aspects of the work in ensuring that questions related to the accuracy or integrity of any part of the work are appropriately investigated and resolved. All animal experiments were performed under a project license (No. 2018-1327) granted by Institute Animal care and Use Committee of The Second Hospital of Shanxi Medical University for the care and use of animals.

Open Access Statement: This is an Open Access article distributed in accordance with the Creative Commons Attribution-NonCommercial-NoDerivs 4.0 International License (CC BY-NC-ND 4.0), which permits the noncommercial replication and distribution of the article with the strict proviso that no changes or edits are made and the original work is properly cited (including links to both the formal publication through the relevant DOI and the license). See: https://creativecommons.org/licenses/by-nc-nd/4.0/.

\section{References}

1. Cooper LT Jr. Myocarditis. N Engl J Med 2009;360:1526-38.

2. Yajima T, Knowlton KU. Viral myocarditis: from the perspective of the virus. Circulation 2009;119:2615-24.

3. Weithauser A, Bobbert P, Antoniak S, et al. Proteaseactivated receptor- 2 regulates the innate immune response to viral infection in a coxsackievirus $\mathrm{B} 3$-induced myocarditis. J Am Coll Cardiol 2013;62:1737-45.

4. Esfandiarei M, McManus BM. Molecular biology and pathogenesis of viral myocarditis. Annu Rev Pathol 2008;3:127-55.

5. Cai Z, Shen L, Ma H, et al. Involvement of Endoplasmic Reticulum Stress-Mediated C/EBP Homologous Protein Activation in Coxsackievirus B3-Induced Acute Viral Myocarditis. Circ Heart Fail 2015;8:809-18.

6. Bao JL, Lin L. MiR-155 and miR-148a reduce cardiac injury by inhibiting NF-kappaB pathway during acute viral myocarditis. Eur Rev Med Pharmacol Sci 2014;18:2349-56.

7. Miyawaki A, Obana M, Mitsuhara Y, et al. Adult murine cardiomyocytes exhibit regenerative activity with cell cycle reentry through STAT3 in the healing process of myocarditis. Sci Rep 2017;7:1407.

8. Divakaran V, Mann DL. The emerging role of microRNAs in cardiac remodeling and heart failure. Circ Res 2008;103:1072-83.

9. Viereck J, Thum T. Long Noncoding RNAs in Pathological Cardiac Remodeling. Circ Res 2017;120:262-4.

10. Corsten MF, Heggermont W, Papageorgiou AP, et al. The microRNA-221/-222 cluster balances the antiviral and inflammatory response in viral myocarditis. Eur Heart J 2015;36:2909-19.

11. Perry RB, Ulitsky I. The functions of long noncoding RNAs in development and stem cells. Development 2016;143:3882-94.

12. Adams BD, Parsons C, Walker L, et al. Targeting noncoding RNAs in disease. J Clin Invest 2017;127:761-71.

13. Chen D, Wu L, Liu L, et al. Comparison of HIF1A AS1 and HIF1A AS2 in regulating HIF $1 \alpha$ and the osteogenic differentiation of PDLCs under hypoxia. Int J Mol Med 
2017;40:1529-36.

14. Li Y, Yang N. Microarray Expression Profile Analysis of Long Non-Coding RNAs in Thoracic Aortic Aneurysm. Kaohsiung J Med Sci 2018;34:34-42.

15. Westermann D, Savvatis K, Lindner D, et al. Reduced degradation of the chemokine MCP-3 by matrix metalloproteinase-2 exacerbates myocardial inflammation in experimental viral cardiomyopathy. Circulation 2011;124:2082-93.

16. He Q, Tan J, Yu B, et al. Long Noncoding RNA HIF1AAS1A Reduces Apoptosis of Vascular Smooth Muscle Cells: Implications for the Pathogenesis of Thoracoabdominal Aorta Aneurysm. Pharmazie 2015;70:310-5.

17. Wu Y, Ding J, Sun Q, et al. Long Noncoding RNA Hypoxia-Inducible Factor 1 Alpha-Antisense RNA 1 Promotes Tumor Necrosis Factor- $\alpha$-Induced Apoptosis Through Caspase 3 in Kupffer Cells. Medicine (Baltimore) 2018;97:e9483.

18. Zhang QQ, Xu MY, Qu Y, et al. TET3 Mediates the Activation of Human Hepatic Stellate Cells via Modulating the Expression of Long Non-Coding RNA HIF1A-AS1. Int J Clin Exp Pathol 2014;7:7744-51.

19. Xue X, Luo L. LncRNA HIF1A-AS1 Contributes to Ventricular Remodeling After Myocardial Ischemia/ Reperfusion Injury by Adsorption of microRNA-204 to Regulating SOCS2 Expression. Cell Cycle 2019;18:2465-80.

20. van den Hoogen P, van den Akker F, Deddens JC, et al. Heart Failure in Chronic Myocarditis: A Role for microRNAs? Curr Genomics 2015;16:88-94.

21. Mercer TR, Dinger ME, Mattick JS. Long non-

Cite this article as: Cao H, Yang B, Zhao Y, Deng X, Shen $\mathrm{X}$. The pro-apoptosis and pro-inflammation role of LncRNA HIF1A-AS1 in Coxsackievirus B3-induced myocarditis via targeting miR-138. Cardiovasc Diagn Ther 2020;10(5):12451255. doi: $10.21037 / \mathrm{cdt}-20-545$ coding RNAs: insights into functions. Nat Rev Genet 2009;10:155-9.

22. Heymans S, Eriksson U, Lehtonen J, et al. The Quest for New Approaches in Myocarditis and Inflammatory Cardiomyopathy. J Am Coll Cardiol 2016;68:2348-64.

23. Viereck J, Kumarswamy R, Foinquinos A, et al. Long noncoding RNA Chast promotes cardiac remodeling. Sci Transl Med 2016;8:326ra22.

24. Wang Z, Zhang XJ, Ji YX, et al. The long noncoding RNA Chaer defines an epigenetic checkpoint in cardiac hypertrophy. Nat Med 2016;22:1131-9.

25. Jiang F, Zhou X, Huang J. Long Non-Coding RNA-ROR Mediates the Reprogramming in Cardiac Hypertrophy. PLoS One 2016;11:e0152767.

26. Wang Y, Liang J, Xu J, et al. Circulating Exosomes and Exosomal lncRNA HIF1A-AS1 in Atherosclerosis. Int J Clin Exp Pathol 2017;10:8383-8.

27. Acun A, Zorlutuna P. Engineered Myocardium Model to Study the Roles of HIF- $1 \alpha$ and HIF1A-AS1 in ParacrineOnly Signaling Under Pathological Level Oxidative Stress. Acta Biomater 2017;58:323-36.

28. Wang J, Chen L, Li H, et al. Clopidogrel Reduces Apoptosis and Promotes Proliferation of Human Vascular Endothelial Cells Induced by Palmitic Acid via Suppression of the Long Non-Coding RNA HIF1A-AS1 in Vitro. Mol Cell Biochem 2015;404:203-10.

29. He Z, Yang D, Fan X, et al. The Roles and Mechanisms of lncRNAs in Liver Fibrosis. Int J Mol Sci 2020;21:1482.

30. Peng H, Wan LY, Liang JJ, et al. The Roles of lncRNA in Hepatic Fibrosis. Cell Biosci 2018;8:63. 